\title{
Verifying Temporal Properties without Temporal Logic
}

\author{
BOWEN ALPERN
}

IBM T. J. Watson Research Center

and

FRED B. SCHNEIDER

Cornell University

An approach to proving temporal properties of concurrent programs that does not use temporal logic as an inference system is presented. The approach is based on using Buchi automata to specify properties. To show that a program satisfies a given property, proof obligations are derived from the Buchi automata specifying that property. These obligations are discharged by devising suitable invariant assertions and variant functions for the program. The approach is shown to be sound and relatively complete. A mutual exclusion protocol illustrates its application.

Categories and Subject Descriptors: D.2.1 [Software Engineering]: Requirements/Specificationsmethodologies; D.2.4 [Software Engineering]: Program Verification-correctness proofs; D.3.1 [Programming Languages]: Formal Definitions and Theory-semantics; F.3.1 [Logics and Meanings of Programs]: Specifying and Verifying and Reasoning about Programs

General Terms: Languages, Theory, Verification

Additional Key Words and Phrases: Assertional reasoning about programs, Buchi automata, liveness properties, program verification, proving temporal logic, safety properties

\section{INTRODUCTION}

Experience has shown that, while it may be possible to convince oneself of the correctness of a sequential program by considering some subset of its executions, this is impossible for concurrent programs. Consequently, methods have been devised to deduce properties of program behavior from the program text itself. The program text obviously contains all the information needed to decide what

This work was supported in part by the Office of Naval Research under contract N00014-86-K-0092, the National Science Foundation under grants DCR-8320274 and CCR-8701103, and the Digital Equipment Corporation. Any opinions, findings, and conclusions or recommendations expressed in this publication are those of the authors and do not reflect the views of these sponsors.

Some of the results in this paper were first presented at the "IEEE Symposium on Logic in Computer Science," Ithaca, New York, June 1987.

Authors' addresses: B. Alpern, IBM 'T. J. Watson Research Center, P.O. Box 218, Yorktown Heights, NY 10598; and F. B. Schneider, Department of Computer Science, Cornell University, Ithaca, NY 14853.

Permission to copy without fee all or part of this material is granted provided that the copies are not made or distributed for direct commercial advantage, the ACM copyright notice and the title of the publication and its date appear, and notice is given that copying is by permission of the Association for Computing Machinery. To copy otherwise, or to republish, requires a fee and/or specific permission.

(C) 1989 ACM 0764-0925/89/0100-0147 $\$ 01.50$ 
executions are possible. Moreover, although the number of possible executions is likely to be very large, only a single program text need be analyzed.

An execution of a program can be viewed as an infinite sequence of states called a history. In a history, the first state is an initial state of the program and each following state results from executing a single atomic action in the preceding state. Terminating executions are extended to infinite histories by repeating the final state. In a concurrent or distributed program, a history is the sequence of states that results from the interleaved execution of atomic actions of the processes.

A property is a set of sequences of states; a program satisfies a property if each of its histories is in the property. Specifying a property as a predicate on sequences allows the essence of that property to be made explicit. Formulas of temporal logic can be interpreted as predicates on sequences of states, and various formulations of such temporal logics have been used for specifying propertiescalled temporal properties - of interest to designers of concurrent programs [15, $16,21,35$ ]. While there is not general agreement on the details of such a specification language, there is agreement that temporal logic provides a good basis for such a language, and it, or something close to it, is sufficiently expressive.

Temporal logic has also been used in proving temporal properties of concurrent programs $[22,25,29,31]$. Here, a program is regarded as defining a collection of temporal logic axioms. The programmer proves that a program satisfies some property of interest by using these axioms along with program-independent axioms and inference rules of temporal logic [23] to show that the temporal formula characterizing the property is a theorem of the logic. Thus, proving that a program satisfies a property is reduced to theorem proving in a temporal logic.

This paper describes a different approach for proving temporal properties of (concurrent) programs. The approach is based on specifying a property as a Boolean combination of deterministic Buchi automata. Proof obligations are extracted from these automata. These obligations generalize the invariant and variant function used to prove partial correctness and termination of sequential programs and define verification conditions that must hold for any program satisfying the property. The verification conditions themselves can be formulated as Hoare triples [11], so reasoning in temporal logic is not required.

We proceed as follows: Section 2 gives the semantics of the programs that we consider. Section 3 reviews Buchi automata and explains how they can be used to specify properties. Extraction of proof obligations from Buchi automata is discussed in Section 4. Section 5 illustrates our method on a mutual exclusion protocol. Section 6 compares our approach to related work, and Section 7 is a summary.

\section{PROGRAMS}

A program $\Pi$ is specified by

$-S_{\mathrm{n}}$, a countable set of program states;

-Init $\subseteq S_{\Pi}$, a set of possible initial states; and

$-\mathscr{A}_{\mathrm{n}}$, a finite set of atomic actions.

An atomic action defines a set of pairs of program states and is therefore a subset of $S_{\mathrm{II}} \times S_{\Pi}$. Atomic action $\alpha$ is enabled in a state $s$ provided ( $\exists t:\langle s, t\rangle$ 


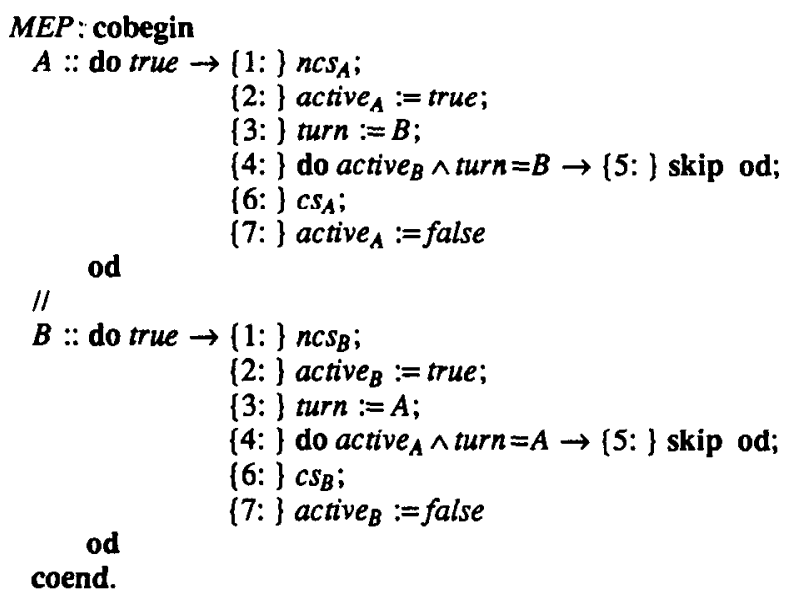

Fig. 1. Peterson's mutual exclusion protocol.

$\in \alpha)$. The statement

$$
\text { (if } b \rightarrow C \text { fì }
$$

is used to specify an atomic action containing those elements $\langle s, t\rangle$ such that (predicate) $b$ holds on $s$ and $t$ is the state produced by executing (assignment) $C$ starting in state $s$.

A program is usually presented as a text, where statements or phrases in the text denote atomic actions. Rather than enumerating the atomic action of a program directly, it is frequently convenient to identify in such a text the control points that delimit atomic actions. In this paper, control points are denoted by marking and numbering them and an atomic action is described by the text between these marks. For example, program fragment of process $A$,

$$
\ldots\{3:\} x:=23\{4\} \ldots,
$$

defines a single atomic action

$$
\alpha_{3}:\left\langle\text { if } p c_{A}=3 \rightarrow p c_{A}:=4 ; x:=23 \mathbf{f i}\right\rangle
$$

where $p c_{A}$ simulates the program counter for process $A$. Thus, $\alpha_{3}$ is enabled in any state in which $p c_{A}=3$.

Formally, a history of a program $\Pi$ is any sequence of states from $S_{\text {II }}$ such that the first state is in Init $t_{\Pi}$ and every subsequent state is the result of executing an enabled atomic action from $\mathscr{X}_{\Pi}$ on the previous state in the sequence. Notice that no restriction is made about the choice of an atomic action when more than one is enabled.' ${ }^{1}$ To ensure that all histories are infinite, we include in $\mathscr{A}_{\Pi}$ an atomic action that has no affect on the program state and is enabled when no other atomic action is.

An example program $M E P$ is shown in Figure 1. It is a simplified version of the solution to the two-process critical section problem described in [30]. ${ }^{2}$ Based

\footnotetext{
${ }^{1}$ Fairness is discussed in Section 5.2.

${ }^{2}$ To simplify the presentation, we have assumed that the noncritical section (ncs) always terminates. However, the algorithm and our proof do not rely on this assumption.
} 


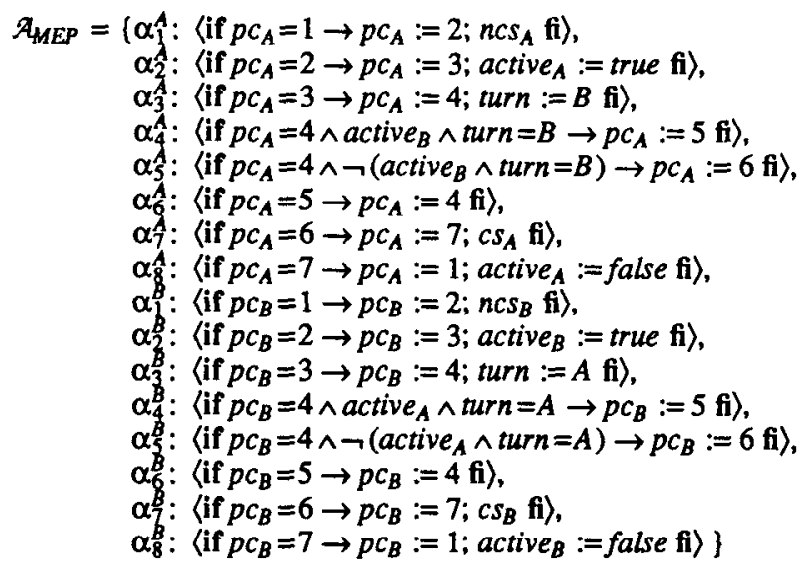

Fig. 2. Atomic actions $\mathscr{X}_{M E P}$.

on the control point annotations, we obtain the set of atomic actions $\mathscr{A}_{M E P}$ of Figure 2. In those atomic actions, variable $p c_{A}$ simulates the program counter for process $A$ and $p c_{B}$ simulates the program counter for process $B$. Finally, we have

$$
\text { Init }_{M E P}=\left\{s \mid s \in S_{M E P} \wedge s \vDash\left(p c_{A}=p c_{B}=1 \wedge(\text { turn }=A \vee \text { turn }=B)\right)\right\}
$$

because, when execution is begun, both processes start at the beginning of their loops and turn is initialized.

\section{SPECIFYING PROPERTIES USING BUCHI AUTOMATA}

A property is a set of infinite sequences of program states. We restrict attention to properties that can be specified by formulas of some linear-time, temporal logic with first-order monadic predicates-that is, formulas composed of temporal operators, Boolean connectives, and atoms that are first-order predicates of the program states. Such logics are slightly more expressive than propositional temporal logics where the atoms are propositions. However, our temporal formulas can be treated as if they were propositional temporal formulas over different sequences. The elements of these sequences are the equivalence classes of the program states under the monadic predicates.

A Buchi automaton is a finite-state machine that accepts or rejects infinite sequences of input symbols [6]. Such an automaton $m$ can be used to specify the property containing those sequences of program states accepted by $m$. Procedures exist to translate propositional temporal formulas into Buchi automata where automaton state transitions are defined in terms of the atoms of the temporal formula [1, 8]. Therefore, restricting consideration to properties that can be specified by Buchi automata-as we do in this paper-is not an additional restriction. Moreover, Buchi automata have natural diagrammatic representations, and this is sometimes a convenient way to specify a property.

An example of a Buchi automaton $m_{a e}$ is given in Figure 3. It accepts infinite sequences in which after a finite prefix each state satisfies the program-state predicate $p$. In temporal logic, this property is specified as $\diamond \square p$. Automaton $m_{a e}$ contains three automaton states labeled $q_{0}, q_{1}$, and $q_{2}$. The start state, $q_{0}$, is 


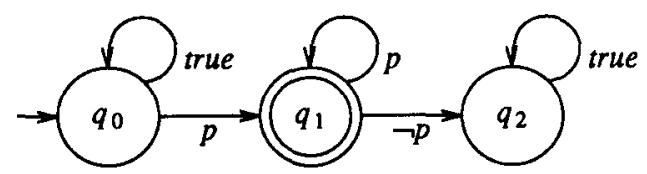

Fig. 3. $m_{a c}$.

denoted by an edge with no origin, and accepting state, $q_{1}$, is denoted by two concentric circles. A Buchi automaton accepts a sequence $\sigma$ iff it enters an accepting state infinitely often while reading $\sigma$ (assuming nondeterministic choices are resolved in favor of acceptance). Notice that there is no way in $m_{a e}$ to get from $q_{2}$ to an accepting state. Such states are called dead states. If an automaton is in a dead state, it cannot accept its input.

Edges between automaton states are labeled by program-state predicates that are called transition predicates and define transitions between automaton states. If a program state satisfies the transition predicate on an edge, then the edge is defined for that program state. For example, because there is an edge labeled $p$ from $q_{0}$ to $q_{1}$ in $m_{a e}$, whenever $m_{a e}$ is in $q_{0}$ and the next symbol read is a program state satisfying $p$, then a transition to $q_{1}$ can be made. We adopt the convention that there be at least one edge defined from each automaton state for each input symbol.

In order to define a Buchi automaton formally, the following notation will be useful: For any sequence $\sigma=s_{0} s_{1} \ldots$,

$$
\begin{aligned}
\sigma[i] & =s_{i} \\
\sigma[\ldots]] & \equiv s_{0} s_{1} \cdots s_{i-1}, \\
\sigma[i . .] & \equiv s_{i} s_{i+1} \ldots, \\
|\sigma| & \equiv \text { the length of } \sigma(\omega \text { if } \sigma \text { is infinite }), \\
I N F(\sigma) & \equiv\{s \mid s \text { appears infinitely often in } \sigma\} .
\end{aligned}
$$

A Buchi automaton $m$ for a property of a program $\Pi$ is a five-tuple $\left\langle S_{\Pi}, Q, Q_{0}\right.$, $A, \delta\rangle$, where

$$
\begin{aligned}
S_{\Pi} & \text { is the (countable) } \text { set }^{3} \text { of program states of } \Pi, \\
Q & \text { is the (finite) set of automaton states of } m, \\
Q_{0} \subseteq Q & \text { is the set of start states of } m, \\
A \subseteq Q & \text { is the set of accepting states of } m, \\
\delta \in(Q \times S) \rightarrow 2^{Q}-\varnothing & \text { is the transition function of } m .
\end{aligned}
$$

Transition function $\delta$ can be extended to handle finite sequences of program states in the usual way:

$$
\delta^{*}(q, \sigma) \equiv \begin{cases}\{q\} & \text { if } \quad|\sigma|=0 \\ \left\{q^{\prime} \mid \exists q^{\prime \prime}: q^{\prime \prime} \in \delta^{*}(q, \sigma[\ldots|\sigma|-1]):\right. & \text { if } 0<|\sigma|<\omega \\ \left.q^{\prime} \in \delta\left(q^{\prime \prime}, \sigma[|\sigma|-1]\right)\right\} & \text { if }\end{cases}
$$

${ }^{3}$ Technically, this is the finite set of equivalence classes (under the monadic predicates of the temporal formula being specified) of program states. 
Fig. 4. $m_{i 0}$.

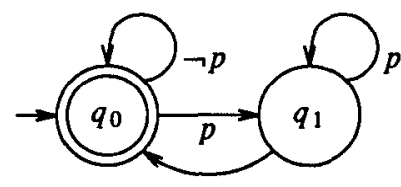

$\neg p$

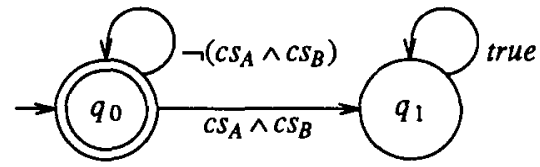

Fig. 5. $m_{\text {mutex }}$

It is often convenient to represent $\delta$ by using transition predicates, as in the diagram above. The transition predicate $t_{i j}$ associated with the edge from automaton state $q_{i}$ to $q_{j}$ holds for a program state $s$ iff $q_{j} \in \delta\left(q_{i}, s\right)$.

A sequence of automaton states that $m$ might occupy while reading $\sigma$ is called a run. Thus, $\rho$ is a run of $m$ on $\sigma$ iff

$$
\rho[0] \in Q_{0} \quad \text { and } \quad(\forall i: 0<i<|\sigma|: \rho[i] \in \delta(\rho[i-1], \sigma[i-1])) .
$$

A Buchi automaton $m$ accepts a sequence $\sigma$ iff there is a run $\rho$ of $m$ on $\sigma$ for which $I N F(\rho) \cap A \neq \varnothing$.

Notice in $m_{a e}$ (Figure 3) that two transitions are possible from $q_{0}$ for a program state satisfying $p$, because any program state that satisfies $p$ also satisfies true. When there is more than one start state or more than one transition is possible from some automaton state for a given input symbol, the automaton is nondeterministic; otherwise, it is deterministic. Thus, $m_{a e}$ is nondeterministic. Using a nondeterministic Buchi automaton, it is possible to specify a property that cannot be specified by a single deterministic Buchi automaton. However, any property that is specified by a nondeterministic automaton can be specified as a Boolean combination of properties, each of which can be specified by a deterministic Buchi automaton. For example, $\diamond \square p$, the property specified by $m_{a e}$, is the negation of the property $\square \diamond \neg p$, specified by the deterministic Buchi automaton $m_{i 0}$ of Figure 4.

\subsection{Examples of Property Specifications}

A Buchi automaton $m_{\text {mutex }}$ that specifies the property of Mutual Exclusion for two processes is given in Figure 5. Mutual Exclusion is the set of sequences in which there is no state where the program counters for two or more processes denote control points inside critical sections. In $m_{\text {mutex }}$, we assume $c s_{A}\left(c s_{B}\right)$ holds for any state in which process $A(B)$ is executing in its critical section.

The property Partial Correctness for pre and post includes all sequences of program states where, if the first state in the sequence satisfies pre, then, in any state where the program counter denotes the end of the program, post is satisfied. A Buchi automaton $m_{p c}$ that specifies this property is shown in Figure 6. In it, done is a predicate that holds for program states in which the program counter denotes the end of the program. 


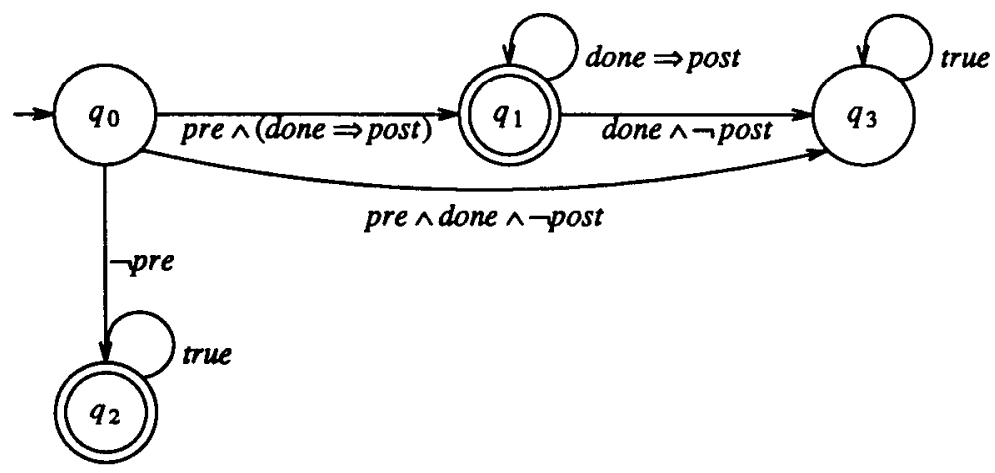

Fig. 6. $m_{p r}$.

\section{PROOF OBLIGATIONS}

Every temporal property $\mathscr{P}$ is a Boolean combination of properties that can be specified by deterministic Buchi automata. Without loss of generality, we assume this combination is in conjunctive normal form. Thus, $\mathscr{P}$ is the conjunction of clauses $\mathscr{P}_{1}, \mathscr{P}_{2}, \ldots, \mathscr{P}_{m}$. To prove that a program $\Pi$ satisfies $\mathscr{P}$, one proves separately that the program satisfies each of these clauses. This establishes that every history of program $\Pi$ is in the property (i.e., set of sequences of program states) specified by each clause, so we can conclude that every history of $\Pi$ is in the intersection of the properties specified by the clauses and that the program satisfies $\mathscr{P}$. Thus, it suffices to derive proof obligations for a single clause.

The proof obligations for a single clause involve exhibiting three proof instruments. The first proof instrument, an invariant, handles the safety aspects of the proof; the second, a variant function, handles the liveness aspects; and the third, a candidate function, arbitrates among the automata specifying the disjuncts of a clause to ensure that any program execution will be accepted by at least one automaton representing a deterministic property or rejected by at least one automaton representing the negation of a deterministic property. The three instruments define verification conditions, which can be formulated as Hoare triples.

\subsection{Proof Instruments}

Given a clause $\mathscr{P}_{i}$ of the form

$$
\mathscr{D}_{1} \vee \ldots \vee \mathscr{D}_{p} \vee \neg \mathscr{D}_{p+1} \vee \ldots \vee \neg \mathscr{D}_{p+n}
$$

where each $\mathscr{D}_{k}$ is specified by deterministic Buchi automaton $m_{k}=\left\langle S_{\mathrm{II}}, Q_{k},\left\{q_{k 0}\right\}\right.$, $\left.A_{k}, \delta_{k}\right\rangle$, we call $m_{1}$ through $m_{p}$ the positive automata of $\mathscr{P}_{i}$ and $m_{p+1}$ through $m_{p+n}$ the negative automata.

To formulate proof instruments for program $\Pi$ and clause $\mathscr{P}_{i}$, define a joint state $x$ to be an element of $J S\left(\mathscr{P}_{i}, \Pi\right)$, the joint state space, $Q_{1} \times \ldots \times Q_{p+n} \times$ $S_{\mathrm{\Pi}}$. Let $x^{(k)}$ denote the $k$ th component of a joint state $x$, and let $x^{(\mathrm{I})}$ abbreviate $x^{(p+n+1)}$. A joint state $x$ is positive, denoted $\operatorname{Pos}(x)$, iff for some positive automaton $m_{j}, x^{(j)} \in A_{j}$. Thus, $x$ is positive if one of $m_{1}$ through $m_{p}$ is in an 
accepting state. The set of negative automata that are in an accepting state in a joint state $x$ is neg $(x)=\left\{k \mid p<k \leq p+n \wedge x^{(k)} \in A_{k}\right\}$. For $\rho$, a sequence of joint states, define $\rho^{(k)}$ to be the projection of $\rho$ onto its $k$ th element; that is, $\rho^{(k)}=$ $\rho[0]^{(k)} \rho[1]^{(k)}$... A sequence of joint states $\rho$ is a joint history of II and $\mathscr{P}_{i}$ iff $\rho^{(\Pi)}$ is a history of $\Pi$ and for all $1 \leq k \leq p+n, \rho^{(k)}$ is a run of $m_{k}$ on $\rho^{(\Pi)}$.

A program $\Pi$ satisfies the property specified by a clause $\mathscr{P}_{i}$ iff each history $\sigma$ of $\Pi$ is either accepted by one of the positive automata-and therefore satisfies one of the properties $\mathscr{D}_{1}$ through $\mathscr{D}_{p}$-or is rejected by one of the negative automata-and therefore satisfies one of $\neg \mathscr{D}_{p+1}$ through $\neg \mathscr{D}_{p+n}$. Thus, $\Pi$ satisfies $\mathscr{P}_{i}$ iff for every history $\sigma$ there is a joint history $\rho$ such that $\sigma=\rho^{(\mathrm{I})}$ and either (1) there is a positive automaton $m_{j}$, such that $I N F\left(\rho^{(j)}\right) \cap A_{j} \neq \varnothing$, or (2) there is a negative automaton $m_{k}$, such that $\operatorname{INF}\left(\rho^{(k)}\right) \cap A_{k}=\varnothing$.

To prove that $\Pi$ satisfies the property specified by a clause $\mathscr{P}_{i}$, three proof instruments must be exhibited:

(1) an invariant $I \subseteq J S\left(\mathscr{P}_{i}, \Pi\right)$;

(2) a candidate function $u: J S\left(\mathscr{P}_{i}, \Pi\right) \rightarrow 2^{[p+1 . . p+n]}$; and

(3) a variant function $v: J S\left(\mathscr{P}_{i}, \Pi\right) \rightarrow W$, where $W$ is a well-founded set. ${ }^{4}$

The invariant relates program states in a history to automaton states occupied while reading that history. The candidate function identifies negative automata that might never again enter an accepting state. The variant function bounds the number of times that the candidate function can become empty before one of the positive automata enters an accepting state.

If the invariant, candidate function, and variant function satisfy the obligations below, then $\Pi$ will satisfy $\mathscr{P}_{i}$. In these obligations, $x$ and $y$ denote elements of $J S\left(\mathscr{P}_{i}, \Pi\right)$, and predicate $x \rightarrow y$ abbreviates

$$
x \in I \wedge \bigwedge_{1 \leq i \leq n+p} \delta_{i}\left(x^{(i)}, x^{(\Pi)}\right)=y^{(i)} \wedge\left(\exists \alpha: \alpha \in \mathscr{A}_{\Pi}:\left\langle x^{(\Pi)}, y^{(\Pi)}\right\rangle \in \alpha\right) .
$$

The obligations are as follows:

O1: For all $x \in J S\left(\mathscr{P}_{i}, \Pi\right),\left(\bigwedge_{1 \leq i \leq n+p} x^{(i)}=q_{i 0} \wedge x^{(\mathrm{II})} \in\right.$ Init $\left._{\Pi}\right) \Rightarrow x \in I$.

02: For all $x, y \in J S\left(\mathscr{P}_{i}, \Pi\right), x \rightarrow y \Rightarrow y \in I$.

O3: For all $x, y \in J S\left(\mathscr{P}_{i}, \Pi\right), x \rightarrow y \Rightarrow(\operatorname{Pos}(y) \vee v(x) \geq v(y))$.

O4: For all $x, y \in J S\left(\mathscr{P}_{i}, \Pi\right), x \rightarrow y \Rightarrow(\operatorname{Pos}(y) \vee v(x)>v(y) \vee \varnothing \subset u(y) \subseteq$ $u(x)-n e g(y))$.

The first two obligations ensure that $I$ holds throughout any joint history. 01 requires that the first state of a joint history be in $I ; 02$ requires that, if one state in a joint history is in $I$, then so must the next.

The third proof obligation requires that variant function $v$ increase only upon entering a positive state. It ensures that either (1) some positive automaton enters an accepting state infinitely often (and the history is accepted), or (2) after some point, $v$ never increases and no positive automaton subsequently enters an accepting state.

\footnotetext{
${ }^{4}$ Recall that a set $W$ is well founded iff it does not contain an infinite descending sequence of elements.
}

ACM Transactions on Programming Languages and Systems, Vol. 11, No. 1, January 1989. 
The final proof obligation, 04, ensures that a history not accepted by a positive automaton is rejected by some negative automaton. To see how, observe that, after some initial prefix of a history that is not accepted by a positive automaton, there will be no positive state, and $v$ will be constant (since its range is well founded). In this case, $\mathrm{O} 4$ requires that, after executing an atomic action, the candidate function on the new state:

- contain only negative automata that were in the candidate function on the old state,

- not contain automata that are accepting in the new state, and

- not be empty.

Thus, unless a positive automaton accepts a history, there must be some negative automaton that, after some point in the history, is thereafter in the candidate function. This automaton rejects the history since it cannot be in an accepting state after the prefix.

We can now prove the following theorem:

SOUNDNESS THEOREM. If there are proof instruments $I, v$, and $u$ that satisfy obligations 01-04, then program $\Pi$ satisfies property $\mathscr{P}_{i}$.

Proof. Let $\rho$ be a joint history of $\Pi$ and $\mathscr{P}_{i} \cdot \rho[0] \in I$ by construction (01). By 02 and induction, $\rho[j] \in I$ for all $0<j$. We must show that $\rho$ causes some positive automaton to be in an accepting state infinitely often or some negative automaton to be in an accepting state only finitely often. Assume that no positive automaton is in an accepting state infinitely often. Thus, there is an index $l_{1}$ such that $\rho\left[l_{1} ..\right]$ contains no positive states. By $\mathrm{O} 3$, the variant function is nonincreasing on $\rho\left[l_{1} ..\right]$. Since its range is well founded, there must be an index $l_{2}$ such that $l_{1} \leq l_{2}$ and the variant function is constant on $\rho\left[l_{2} ..\right]$. By 04, there is a negative automaton $m_{k}$ such that, for all $j>l_{2}$,

$$
k \in u(\rho[j]) \wedge k \notin n e g(\rho[j]) .
$$

Therefore, $m_{k}$ does not enter an accepting state after $l_{2}$. This means that $m_{k}$ rejects $\rho$, so $\Pi$ satisfies $\mathscr{P}_{i}$.

We now show that the method is relatively complete.

COMPLETENESS THEOREM. If program $\Pi$ satisfies property $\mathscr{P}_{i}$, then there exist proof instruments $I, v$, and $u$ that satisfy obligations $01-04$.

Proof. Form a directed graph where the nodes are the joint states and there is an edge from node $x$ to $y$ iff $y$ is not a positive accepting state and $x$ immediately precedes $y$ in some joint history. Define transitive, antisymmetric, relation $\gg$ on the nodes of this graph such that $x \gg y$ iff $x \neq y$, there is a path from $x$ to $y$, and an accepting state for each negative automaton appears somewhere on this path.

Relation $\gg$ is well founded, as is shown by the following proof by contradiction: If $\gg$ were not well founded, then there would be an infinite descending chain $x_{1}$ $\gg x_{2} \ldots$ By construction of the graph, this implies the existence of a joint history that includes $x_{1}$. Let $\sigma_{0}$ be a prefix of such a joint history that ends with $x_{1}$. For each $x_{i}$ in the infinite descending chain, let $\sigma_{i}$ be the path from $x_{i}$ to $x_{i+1}$ 
that includes an accepting state for every negative automaton. Such a path exists by definition, because $x_{i} \gg x_{i+1}$. Finally, let $\sigma$ be $\sigma_{0} \sigma_{1} \ldots$ Notice that $\sigma$ is a joint history, that $\sigma$ contains no positive accepting states after $\sigma_{0}$, and that there are infinitely many accepting states for each negative automaton in $\sigma$. Thus, $\sigma$ does not satisfy $\mathscr{P}_{i}$. This is a contradiction, and we conclude that $\gg$ is well founded.

Since $\gg$ is well founded, the following ordinal function is well defined:

$$
H(x)=\sup _{x \rightarrow y}\{H(y)+1\} .
$$

If there is no $y$ such that $x \gg y$, then $H(x)=0$ by definition of sup, so $H$ is total. Notice that if $x \gg y$ then $H(x)>H(y)$. Moreover, if there is any path from $x$ to $y$ in the graph, then $H(x) \geq H(y)$.

The variant function will be constructed using $H$ and the level $l(x)$ of a node $x$, defined as follows: Level $l(x)$ is the largest integer $i$ such that for any collection of $i$ negative automata there exists some node $w$ with $H(w)=H(x)$ such that there is a path from $w$ to $x$ and there is an accepting state for each automaton in the collection somewhere on the path. Note that, by definition of $H$, the level of a node will be less than or equal to $n$. (The equality will hold only if $x$ itself is accepting for all negative automata.)

The three proof instruments can now be defined. Choose $I$ to be the characteristic predicate for the set of joint states that appear in joint histories of $\Pi$ and $\mathscr{P}_{i}$. Choose $v(x)$ to be $\infty$ if $x$ is positive and $\langle H(x), n-l(x)\rangle$ otherwise. The range of $v$ with lexicographic ordering of pairs with $\infty$ larger than any pair is well founded because the ordinals are. Finally, choose $u(x)$ to be the set of negative automata that do not have accepting states on any path to $x$ from any $w$ such that $v(w)=v(x)$. (Note that $u(x)=\varnothing$ only if there is no such $w$ different from $x_{\text {.) }}$

Proof obligations 01 and 02 follow immediately from the definition of $I$.

To see that $\mathrm{O} 3$ holds, notice that if $x \rightarrow y$ and $y$ is not positive then there is a path (of length 1) from $x$ to $y$ in the graph. Thus, $H(x) \geq H(y)$. Suppose $H(x)=H(y)$. Since any path to $x$ can be extended to $y, l(x) \leq l(y)$. Therefore, $v(x) \geq v(y)$.

To see that $\mathrm{O} 4$ holds, suppose that $x \rightarrow y, y$ is not positive, and $v(x)=v(y)$ holds. First, note that neg $(y) \cap u(y)$ is empty, since there is a trivial path from $y$ to itself. Because there is a path from $x$ to $y$, if $m_{k}$ has an accepting state on a path from $w$ to $x$, then $m_{k}$ must have an accepting state on a path from $w$ to $y$. Therefore, $u(y)$ is contained in $u(x)$. Further, since $x \neq y$ holds, $u(y) \neq \varnothing$. 04 follows immediately. This completes the proof.

\subsection{Verification Conditions}

Obligations $01-04$ can be translated into verification conditions formulated as Hoare triples [11] and predicate logic formulas. The Hoare triple $\{P\} \alpha\{Q\}$ for an atomic action $\alpha$ asserts that any execution of $\alpha$ started in a state satisfying $P$ terminates in a state satisfying $Q .{ }^{5}$ Thus, $\{P\} \alpha\{Q\}$ is valid iff

$$
(\forall\langle s, t\rangle:\langle s, t\rangle \in \alpha:(s \vDash P) \Rightarrow(t \vDash Q)) \text {. }
$$

\footnotetext{
${ }^{6}$ Since $\alpha$ is an atomic action, it must terminate once execution commences.

ACM Transactions on Programming Languages and Systems, Vol. 11, No. 1, January 1989.
} 
To reformulate $\mathrm{O} 1-04$ in terms of Hoare triples, we define slight variations of the three proof instruments. Define $P J S\left(\mathscr{P}_{i}\right)$, the projection of $J S\left(\mathscr{P}_{i}, \Pi\right)$ with respect to program states, as $P J S\left(\mathscr{P}_{i}\right)=Q_{1} \times \ldots \times Q_{p+n}$. Elements of $P J S\left(\mathscr{P}_{i}\right)$ are called projected joint states. We write $\bar{x}$ to denote the projection of a joint state $x$. The projected joint state in which every automaton is in its start state is called the projected joint start state and denoted $q_{\overline{0}}$. A projected joint state $\bar{x}$ is considered positive if it is the projection of a positive state. For $\bar{x}$, the projection of $x$ and $s \in S_{\Pi}$, we define the following:

$$
\begin{aligned}
I_{\bar{x}} & \equiv\left\{s \mid s \in S_{\Pi} \wedge\langle\bar{x}, s\rangle \in I\right\}, \\
v_{\bar{x}}(s) & \equiv v(\langle\bar{x}, s\rangle) \\
u_{\bar{x}}(s) & \equiv u(\langle\bar{x}, s\rangle), \\
\operatorname{Pos}_{\bar{x}} & \equiv \operatorname{Pos}(x) \\
n e g_{\bar{x}} & \equiv \operatorname{neg}(x) .
\end{aligned}
$$

Finally, we define a projected joint state transition predicate $t_{\overline{x y}}$ to be a predicate that holds for any program state causing a transition from a projected joint state $\bar{x}$ to a projected joint state $\bar{y} . t_{\overline{x y}}$ can be formed by taking the conjunction over all $m_{i}$ of the transition predicates labeling the edge from $\bar{x}^{(i)}$ to $\bar{y}^{(i)}$.

Satisfying the following two verification conditions implies that 01-04 hold. The first one implies 01:

VC1: Init $_{\Pi} \Rightarrow I_{\overline{0}}$.

The next implies 02-O4:

VC2: For all $\bar{x}, \bar{y} \in P J S\left(\mathscr{P}_{i}\right)$ and $\alpha \in \mathscr{A}_{\mathrm{M}}$,

$$
\begin{aligned}
& \left\{I_{\bar{x}} \wedge v_{\bar{x}}=\mathrm{V} \wedge u_{\bar{x}}=\mathrm{U} \wedge t_{\overline{x y}}\right\} \\
& \alpha \\
& \left\{I_{\bar{y}} \wedge\left(P o s_{\bar{y}} \vee v_{\bar{y}}<\mathrm{V} \vee\left(v_{\bar{y}}=\mathrm{V} \wedge \varnothing \subset u_{\bar{y}} \subseteq \mathrm{U}-n e g_{\bar{y}}\right)\right)\right\} .
\end{aligned}
$$

To see that $\mathrm{O} 2, \mathrm{O} 3$, and $\mathrm{O} 4$ are implied by $\mathrm{VC} 2$, choose

$$
x=\langle\bar{x}, s\rangle \quad \text { and } \quad y=\left\langle\bar{y}, s^{\prime}\right\rangle \text {, }
$$

and assume $x \rightarrow y$. Thus, there is an $\alpha \in \mathscr{A}_{\Pi}$ such that $\left\langle s, s^{\prime}\right\rangle \in \alpha, s \vDash I_{\bar{x}}$, and $s \vDash t_{\overline{x y}}$. Choose $\mathrm{V}$ and $\mathrm{U}$ such that $v_{\bar{x}}(s)=\mathrm{V}$ and $u_{\bar{x}}(s)=\mathrm{U}$ hold. From VC2, we conclude that postcondition

$$
I_{\bar{y}} \wedge\left(P o s_{\bar{y}} \vee v_{\bar{y}}<\mathrm{V} \vee\left(v_{\bar{y}}=\mathrm{V} \wedge \varnothing \subset u_{\bar{y}} \subseteq \mathrm{U}-n e_{\bar{y}}\right)\right)
$$

holds in $y$. However, this is equivalent to

$$
I_{\bar{y}} \wedge\left(P o s_{\bar{y}} \vee v_{\bar{y}} \leq \mathrm{V}\right) \wedge\left(P o s_{\bar{y}} \vee v_{\bar{y}}<\mathrm{V} \vee \varnothing \subset u_{\bar{y}} \subseteq \mathrm{U}-n e_{\bar{y}}\right)
$$

02 then follows from the first conjunct, $\mathrm{O} 3$ from the second, and 04 from the last.

Conversely, assume $\mathrm{O} 1-\mathrm{O} 4$ hold. VC1 follows from $\mathrm{O} 1$. From $\mathrm{O} 2$ we get

$$
\left\{I_{\bar{x}} \wedge t_{\bar{x} \bar{y}}\right\} \alpha\left\{I_{\bar{y}}\right\}
$$

From $\mathrm{O} 3$ we get

$$
\left\{I_{\bar{x}} \wedge v_{\bar{x}}=\mathrm{V} \wedge t_{\overline{x y}}\right\} \alpha\left\{P \operatorname{sos}_{\bar{y}} \vee v_{\bar{y}} \leq \mathrm{V}\right\} .
$$


Finally, from $\mathrm{O} 4$ we get

$$
\left\{I_{\bar{x}} \wedge v_{\bar{x}}=\mathrm{V} \wedge u_{\bar{x}}=\mathrm{U}\right\} \propto\left\{P o s_{\bar{y}} \vee v_{\bar{y}}<\mathrm{V} \vee \varnothing \subset u_{\bar{y}} \subseteq \mathrm{U}-n e g_{\bar{y}}\right\}
$$

Together, these three Hoare triples imply VC2.

We have shown that obligations $01-\mathrm{O} 4$ are equivalent to verification conditions VC1 and VC2. If the underlying assertion language is expressive enough to capture the preconditions and postconditions of the Hoare triples of VC2, then the verification conditions can be expressed in this logic. Since Hoare logic is semantically complete relative to the completeness of the assertion language, our proof technique is complete relative to the semantic and expressive completeness of this logic.

\subsection{Eliminating the Candidate Function}

The invariant and variant functions above are generalizations of standard proof instruments used to prove partial correctness and termination of sequential programs. The candidate function is not standard. It can be a useful proof tool, as illustrated in the example of Section 5.2, but-as we now show-is not necessary for proving temporal properties.

Although the set of properties that can be specified by deterministic Buchi automata is not closed under negation, it is closed under conjunction and disjunction. Thus, any property $\mathscr{P}_{i}$ can be written $\mathscr{M} \vee \neg \mathscr{N}$, where $\mathscr{M}=\mathscr{D}_{1}$ $\vee \ldots \vee \mathscr{D}_{p}$ and is accepted by deterministic Buchi automaton $m_{\mathscr{N}}$, and $\mathscr{N}=$ $\mathscr{D}_{p+1} \wedge \ldots \wedge \mathscr{D}_{p+n}$ and is accepted by deterministic Buchi automaton $m_{\mathscr{N}}$. Having made this observation, the proof obligations for $\mathscr{P}_{i}$ can now be stated without using a candidate function because there exists only one negative automaton. Obligations $01-03$ remain as before. 04 becomes

O4': For all $x, y \in J S\left(\mathscr{P}_{i}, \Pi\right), x \rightarrow y \Rightarrow(\operatorname{Pos}(y) \vee(N e g(y) \Rightarrow v(x)>v(y)))$,

where $\operatorname{Neg}(y)$ holds when $m_{\mathscr{N}}$ is in an accepting state in $y$.

The verification conditions can also be simplified. VC1 is unchanged, and VC2 becomes

VC2': For all $\bar{x}, \bar{y} \in P J S\left(\mathscr{P}_{i}\right)$ and $\alpha \in \mathscr{A}_{\mathrm{II}}$,

$$
\begin{aligned}
& \left\{I_{\bar{x}} \wedge v_{\bar{x}}=\mathrm{V} \wedge t_{\overline{x y}}\right\} \\
& \alpha \\
& \left\{I_{\bar{y}} \wedge\left(P o s_{\bar{y}} \vee\left(\mathrm{V} \geq v_{\bar{y}} \wedge\left(N e g_{\bar{y}} \Rightarrow \mathrm{V}>v_{\bar{y}}\right)\right)\right)\right\},
\end{aligned}
$$

where $N e g_{\bar{y}}$ holds when $m_{N}$ is in an accepting state in $\bar{y}$.

\section{EXAMPLE: PETERSON'S PROTOCOL}

To illustrate our verification method, we prove two properties of Peterson's protocol (Figure 1). First, we prove that it prevents two processes from concurrently executing in critical sections. Then, we prove that a process attempting to enter its critical section will succeed eventually. 


\subsection{Mutual Exclusion}

To prove that the protocol satisfies Mutual Exclusion as specified by $m_{\text {mutex }}$ (Figure 5), we use the following proof instruments:

$$
I=\left\{\left\langle q_{0}, P O L\right\rangle,\left\langle q_{1}, \text { false }\right\rangle\right\},
$$

where

$$
\begin{aligned}
& P O L=(\text { turn }=A \vee \text { turn }=B) \\
& \wedge p c_{A}=3 \Rightarrow \text { active }_{A} \\
& \wedge p c_{A}=4 \Rightarrow \text { active }_{A} \\
& \wedge p c_{A}=5 \Rightarrow \text { active }_{A} \\
& \wedge p c_{A}=6 \Rightarrow \text { active }_{A} \wedge\left(\text { turn }=A \vee \neg \text { active }_{B} \vee p c_{B}=3\right) \\
& \wedge p c_{A}=7 \Rightarrow \text { active }_{A} \wedge\left(\text { turn }=A \vee \neg \text { active }_{B} \vee p c_{B}=3\right) \\
& \wedge p c_{B}=3 \Rightarrow \text { active }_{B} \\
& \wedge p c_{B}=4 \Rightarrow \text { active }_{B} \\
& \wedge p c_{B}=5 \Rightarrow \text { active }_{B} \\
& \wedge p c_{B}=6 \Rightarrow \text { active }_{B} \wedge\left(\text { turn }=B \vee \neg \text { active }_{A} \vee p c_{A}=3\right) \\
& \wedge p c_{B}=7 \Rightarrow \text { active }_{B} \wedge\left(\text { turn }=B \vee \neg a c t i v e_{A} \vee p c_{A}=3\right) \\
& v=0 \\
& u=\varnothing
\end{aligned}
$$

We now show that verification conditions VC1 and VC2 are satisfied with these proof instruments.

To demonstrate $\mathrm{VC} 1$, we must show that $\operatorname{Init}_{\Pi} \Rightarrow I_{0}$ holds, where $I_{0}$ is $P O L$. This is trivial.

To show that VC2 is satisfied, a number of Hoare triples must be checked. For every atomic action $\alpha$, they are

$$
\begin{aligned}
& \left\{I_{\overline{0}} \wedge t_{\overline{00}}\right\} \propto \alpha\left\{I_{\overline{0}}\right\} \\
& \left\{I_{\overline{0}} \wedge t_{\overline{01}}\right\} \propto \alpha\left\{I_{\overline{1}}\right\} \\
& \left\{I_{\overline{1}} \wedge t_{\overline{11}}\right\} \propto\left\{I_{\overline{1}}\right\} .
\end{aligned}
$$

For the automaton of Figure 5,

$$
\begin{aligned}
& c s_{A} \equiv 6 \leq p c_{A} \leq 7 \\
& c s_{B} \equiv 6 \leq p c_{B} \leq 7 .
\end{aligned}
$$

Because

$$
\begin{aligned}
& t_{\overline{00}} \equiv \neg\left(c s_{A} \wedge c s_{B}\right) \\
& t_{\overline{01}} \equiv c s_{A} \wedge c s_{B} \\
& t_{\overline{11}} \equiv \text { true },
\end{aligned}
$$

and $P O L \Rightarrow \neg\left(c s_{A} \wedge c s_{B}\right)$ holds, these Hoare triples simplify to

$$
\begin{aligned}
& \{P O L\} \alpha\{P O L\} \\
& \{\text { false } \alpha\{\text { false }\} \\
& \text { false } \alpha\{\text { false }\}
\end{aligned}
$$


There are 16 atomic actions in the program. Since $\{$ false $\} \alpha\{$ false $\}$ is trivially valid for any $\alpha$, a total of 16 triples must, therefore, be checked. Establishing that these 16 triples are valid can be done by inspection.

Most of the work in this proof concerns invariant $I$ and verifying its invariance. To make this easier to handle, $I$ can be presented as a property outline. A property outline is a program that has been annotated by asserting at each control point the subset of the joint state space in $I$ corresponding to that control point. ${ }^{6}$ Subsets of the joint state space are described by enumeration or by a characteristic predicate, whichever is more convenient. A property outline equivalent to $I$ above is given in Figure 7. Given such a property outline, it is usually possible to verify the triples for $\mathrm{VC} 2$ by inspection.

\subsection{Starvation Freedom and Fairness}

In addition to mutual exclusion, a solution to the critical section problem should ensure that processes attempting entry to critical sections actually do enter eventually. A process of $M E P$ is said to starve if it tries to enter its critical section but never succeeds. Process $A$ of $M E P$ should eventually enter $c s_{A}$ whenever

$$
\operatorname{try}_{A}: p c_{A}=4 \vee p c_{A}=5
$$

holds, ${ }^{7}$ and similarly process $B$ should eventually enter $c s_{B}$ whenever

$$
\operatorname{try}_{B}: p c_{B}=4 \vee p c_{B}=5
$$

holds. The nonstarvation property $\mathscr{N S}_{M E P}$ asserts that neither process starves. This can be formalized in temporal logic as

$$
\mathscr{N S}_{M E P}:\left(\square \diamond \neg \operatorname{try}_{A}\right) \wedge\left(\square \diamond \neg \text { try }_{B}\right) \text {. }
$$

It is easy to see that $M E P$ does not satisfy $\mathscr{N S}_{M E P}$ for some histories in which one or the other process is not given sufficient opportunity to execute. A fairness assumption asserts that an atomic action that is enabled "often enough" will be executed eventually. Let $\mathscr{F}_{\alpha}$ be a fairness assumption for atomic action $\alpha{ }^{8} \mathrm{~A}$ fairness assumption for a program $\Pi$ is the conjunction of fairness assumptionsone for each atomic action in $\mathscr{A}_{11}$. Thus, $\mathscr{T}_{M E P}$-the fairness assumption for $M E P$-is defined by

$$
\mathscr{F}_{M E P}: \bigwedge_{\alpha \in \mathscr{A}_{M E P}} \mathscr{F}_{\alpha}
$$

To show that $M E P$ satisfies $\mathscr{N S}_{M E P}$ for all histories satisfying $\mathscr{F}_{M E P}$, we must prove that $M E P$ satisfies $\mathscr{F}_{M E P} \Rightarrow \mathscr{N S}_{M E P}$. Putting this property into conjunctive normal form, we get

$$
\mathscr{F} \mathcal{N S}_{M E P}:\left(\bigvee_{\alpha \in \mathscr{Q}_{M E P}}^{\vee} \neg \mathscr{F}_{\alpha} \vee \square \diamond \neg t r y_{A}\right) \wedge\left(\bigvee_{\alpha \in \mathscr{Q}_{M E P}}^{\vee} \neg \mathscr{F}_{\alpha} \vee \square \diamond \neg t r y_{B}\right)
$$

\footnotetext{
${ }^{6}$ Proof outlines serve the same purpose in Hoare's logic [17].

${ }^{7}$ This choice of try is stronger than it needs to be, but simplifies the proof.

${ }^{8}$ See [9] for various types of fairness assumptions.

ACM Transactions on Programming Languages and Systems, Vol. 11, No. 1, January 1989.
} 


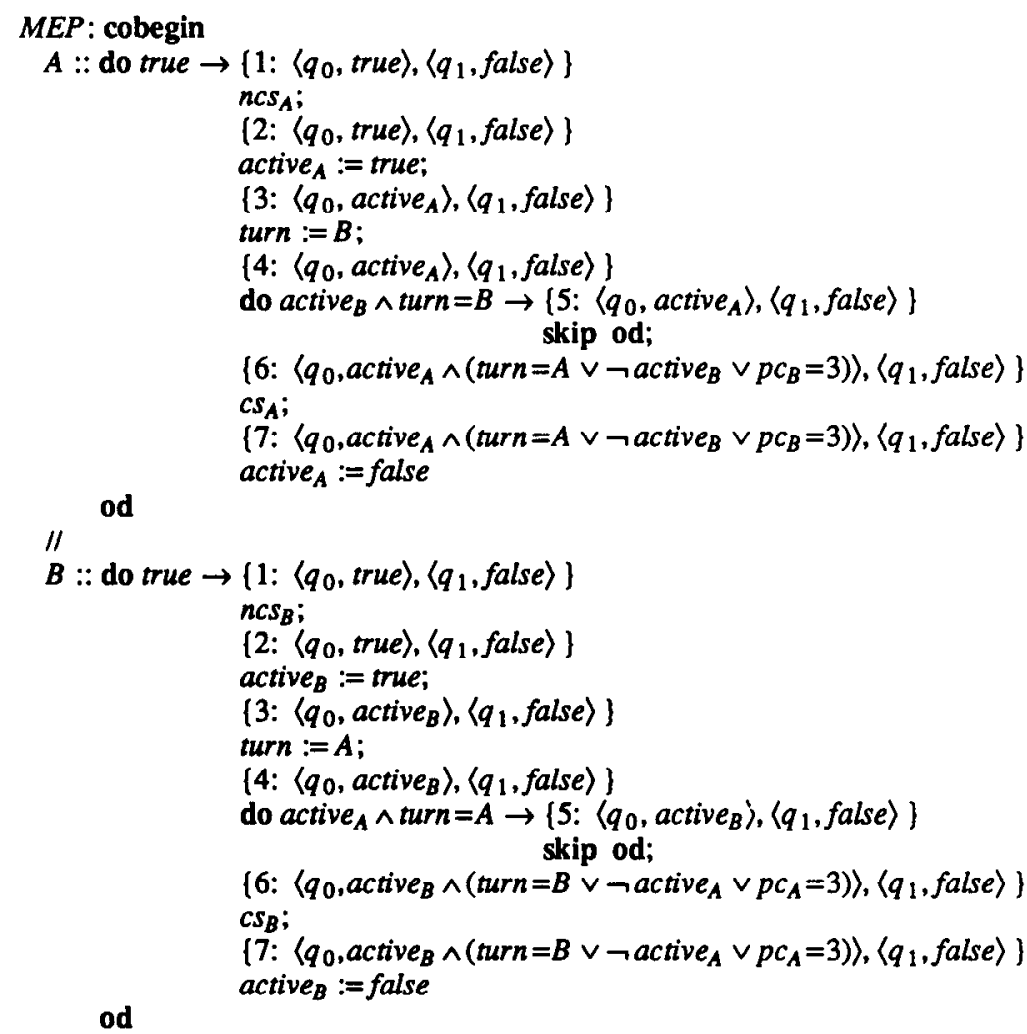

coend.

Fig. 7. Property outline for $I$.

Each of the two clauses (conjuncts) in $\mathscr{F} \mathcal{N S}_{M E P}$ is proved separately. However, since the two clauses are symmetric, only the first one is proved here; the proof of the second is similar.

As a fairness assumption for atomic actions, we choose weak fairness, which asserts that an atomic action that becomes enabled is eventually executed or otherwise becomes disabled. This is expressed in temporal logic as

$$
\mathscr{H Y F}_{\alpha}: \square \diamond \neg \text { enabled }(\alpha) \text {; }
$$

where enabled $(\alpha)$ holds in any program state $s$ where atomic action $\alpha$ is enabled. Thus, the first conjunct of $\mathscr{F} \mathscr{N S}_{M E P}$ is

$$
\begin{aligned}
\mathscr{F} \mathscr{N S}_{M E P}^{A}: & \bigvee_{\alpha \in \mathscr{A}_{M E P}} \neg \mathscr{H F} \mathrm{F} \square \diamond \neg \text { try }_{A} \\
= & \bigvee_{\alpha \in \mathscr{A}_{M E P}} \neg \square \diamond \neg \text { enabled }(\alpha) \vee \square \diamond \neg \text { try }_{A} .
\end{aligned}
$$

This clause contains 17 disjuncts because $\left|\mathscr{A}_{M E P}\right|=16$; all but one of these disjuncts is negated. Each disjunct is of the form $\square \diamond \neg p$, so the Buchi automaton specification for each is $m_{i o}$ of Figure 4. 
To prove that $\mathscr{F} \mathcal{N S}_{M E P}^{A}$ holds, we use the following proof instruments:

$$
\begin{aligned}
& I=J S\left(\mathscr{T N S}_{M E P}, M E P\right) \\
& v= \begin{cases}\infty & \neg \text { try }_{A} \\
p c_{A}-3 & \operatorname{try}_{A} \wedge \text { try }_{B} \wedge \text { turn }=A \\
2+\left(4-p c_{B}\right) \bmod 7 & \operatorname{try}_{A} \wedge \neg \operatorname{try}_{B} \\
7+p c_{B}-3 & \operatorname{try}_{A} \wedge \text { try }_{B} \wedge \text { turn }=B\end{cases} \\
& u= \begin{cases}\varnothing & \neg \text { try }_{A} \\
\left\{i \mid \text { enabled }\left(\alpha_{i}^{A}\right)\right\} & \text { try }_{A} \wedge \operatorname{try}_{B} \wedge \text { turn }=A \\
\left\{i \mid \text { enabled }\left(\alpha_{i}^{B}\right)\right\} & \text { try }_{A} \wedge\left(\neg \text { try } y_{B} \vee \text { turn }=B\right)\end{cases}
\end{aligned}
$$

Rather than checking that verification conditions VC1 and VC2 hold, we argue informally that obligations $\mathrm{O} 1-\mathrm{O} 4$ are satisfied by this choice of proof instruments.

01 and 02 are trivially satisfied by the choice of invariant, since the invariant rules out no joint state.

O3 follows from the construction of the variant function, as follows: When $\neg t r y_{A}$ holds, then an automaton for a positive disjunct is in an accepting state, so $\mathrm{O} 3$ is satisfied in that case. If $\operatorname{try}_{A} \wedge t r y_{B} \wedge$ turn $=A$, then $v$ is the number of atomic actions that $A$ must execute before entering $c s_{A}$. Since turn $=A$, subsequent execution by $B$ does not alter this value. If $\operatorname{try}_{A} \wedge \neg t r y_{B}$, then $v$ includes the number of atomic actions $B$ must execute to establish the previous case (try ${ }_{A}$ $\wedge \operatorname{try}_{B} \wedge$ turn $=A$ ). Clearly, execution by $A$ does not alter this value. Finally, when $t r y_{A} \wedge t r y_{B} \wedge$ turn $=B$, then $v$ is the maximum value of $v$ in the preceding case $\left(\operatorname{try}_{A} \wedge \neg \operatorname{try}_{B}\right.$ ) plus the number of atomic actions that $B$ must execute to make $t r y_{A} \wedge \neg$ try $y_{B}$ hold.

$\mathrm{O} 4$ follows from the construction of the candidate function, which always contains the index of the automaton corresponding to the fairness assumption for the enabled atomic action that will reduce $v$.

Note that this proof is an instance of the method of helpful directions for weak fairness [9]. Each process corresponds to a direction. Execution of an enabled atomic action in the helpful direction decreases the variant function. Execution of an enabled atomic action in some other direction does not increase the variant function, nor does it disable any helpful atomic action. Thus, when $\operatorname{try}_{A} \wedge t r y_{B}$ $\wedge$ turn $=A$, the helpful direction is process $A$; when try $_{A} \wedge\left(\neg\right.$ try $_{B} \vee$ turn $\left.=B\right)$, the helpful direction is process $B$. The weak fairness assumption guarantees that the variant function decreases eventually.

The method of helpful directions is a special case (for proving properties that assume weak fairness) of the technique given in this paper. To see this, observe that the helpful directions identify atomic actions that decrease the variant function. The stipulation that a nonhelpful atomic action not increase the variant function guarantees 03 . The value of the candidate function can be taken to correspond to any subset of the helpful atomic actions. The stipulation that a nonhelpful atomic action leave helpful ones enabled guarantees $\mathrm{O} 4$.

\section{DISCUSSION}

We have shown how to reduce a temporal property into proof obligations that can be formulated as formulas of predicate logic and Hoare's logic. The idea that 
temporal properties can be proved without temporal logic is not new. For example, Manna and Pnueli [20] point out that it is possible to prove temporal properties using a partially interpreted first-order logic with operators that correspond roughly to the right-hand sides of the definitions of the temporal modalities. The use of invariance and well foundedness for proving temporal properties is suggested by Lehmann, Pnueli, and Stavi [18] and by Manna and Pnueli [24]. Manna and Pnueli in [25] advocate using temporal logic along with invariance and well foundedness. What is new in this paper is a systematic method for reducing a temporal property to nontemporal proof obligations.

Other investigations into decomposing temporal properties include [3], [10], [12], [27], [28], and [33]. Most of that work is concerned with decomposing various classes of global temporal properties of a system into local properties of the system components, resulting in so-called compositional proof systems. The work in [10] is most similar to ours in that temporal properties are reduced to primitive formulas that resemble triples. That work, however, is concerned only with finite sequences (both as properties and programs) and therefore does not address the problem we are most concerned with.

Another, related, approach to verifying that a program satisfies a property is model checking $[5,7,19]$. Here, a program $\Pi$ is viewed as specifying a Kripke structure $\mathscr{K}_{\Pi} . \mathscr{K}_{\Pi}$ is a model for a temporal property $\mathscr{P}$ iff $\Pi$ satisfies $\mathscr{P}$. To determine if $\Pi$ satisfies $\mathscr{P}$, it suffices to check whether $\mathscr{K}_{\Pi}$ is a model for $\mathscr{P}$, and this amounts to checking each state in the state space to see which subformulas of $\mathscr{P}$ hold in that state. Thus, for programs with finite-state spaces, it is possible to verify mechanically whether the program satisfies a given temporal property.

Recently, Vardi and Wolper [34] observed that, for programs with finite-state spaces, $\mathscr{H}_{I I}$ can be viewed as a Buchi automaton that accepts exactly the histories of $\Pi$. From this automaton and one that recognizes sequences satisfying $\neg \mathscr{P}$, a Buchi automaton $m_{\Pi \wedge \neg \mathscr{P}}$ can be constructed that accepts all histories of $\Pi$ not satisfying $\mathscr{P}$. The decision procedure for the emptiness problem for $m_{\Pi \wedge \neg \mathscr{P}}$ can then be used to determine if $\Pi$ satisfies $\mathscr{P}$. A similar approach was developed independently by Kurshan [13, 14].

Model checking is restricted to programs with finite-state spaces ${ }^{9}$ but is algorithmic. Since it is algorithmic, it can be mechanized and does not require creativity in devising invariants, variant functions, or candidate functions. Also, model checking is always guaranteed to get the correct answer. In contrast, the methods presented in this paper are not limited to finite-state programs. Unfortunately, the methods are, in general, undecidable. Moreover, they may require creativity in devising suitable proof instruments, although this might be viewed as an asset since the proof instruments can give insight into why a program works.

The first Buchi-automaton-based method for extracting first-order proof obligations for temporal properties was proposed by us in [1] and [2]. That work applied to those properties that can be specified using a single deterministic Buchi automaton. Formulated in the terminology of this paper, the method requires the program prover to exhibit an invariant $I$ and a variant function $v$

\footnotetext{
${ }^{9}$ However, proponents of the model checking approach have made progress in weakening the finitestate assumption so that it applies only to certain key parts of the program $[4,32]$.
} 
satisfying

AS1: For all $x \in J S(\mathscr{P}, \Pi),\left(x^{(1)}=q_{10} \wedge x^{(\Pi)} \in\right.$ Init $\left._{\Pi}\right) \Rightarrow x \in I$;

AS2: For all $x, y \in J S(\mathscr{P}, \Pi), x \rightarrow y \Rightarrow y \in I$; and

AS3: For all $x, y \in J S(\mathscr{P}, \Pi), x \rightarrow y \Rightarrow(\operatorname{Pos}(y) \vee v(y)<v(x))$.

AS1 and AS2 are obligations $\mathrm{O} 1$ and $\mathrm{O} 2$ of the approach outlined in Section 4. Consider the remaining obligations ( 03 and 04 ) of that approach. The property is a single clause that consists of a single, nonnegated property, so there are no negative automata. Thus, $u(x)=\varnothing$ for every joint state $x$, so the final disjunct of $\mathrm{O} 4$ must be false. AS3 and $\mathrm{O} 4$ are therefore equivalent, and each implies $\mathrm{O} 3$. Thus, the two techniques yield essentially the same proof obligations when applied to properties that they both can handle.

The method in [2] is unsatisfactory for properties specified by nondeterministic Buchi automata. To use it to prove that a program $\Pi$ satisfies such a property $\mathscr{P}$, a deterministic property $\mathscr{D}$ that is contained in $\mathscr{P}$ is found. Proof obligations are then extracted from the deterministic Buchi automaton for $\mathscr{D}$. If a (finite-state) program $\Pi$ satisfies $\mathscr{P}$, an appropriate $\mathscr{D}$ always exists, but may be big and difficult to find. Furthermore, the proof obligations for a nondeterministic property now depend on the program as well as on the Buchi automaton for the property to be proved. The approach of Section 4 does not suffer from these difficulties since every property that can be specified using a nondeterministic Buchi automaton can be specified as a Boolean combination of properties specified by deterministic ones [6].

In [26], Manna and Pnueli concurrently and independently developed a different technique for extending the approach in [2] to obtain proof obligations for properties specified by nondeterministic Buchi automata. The approach is based on a $\forall$-automaton for a property. Simplifying slightly, ${ }^{10}$ a $\forall$-automaton is a Buchi automaton that accepts its input iff every run on that input eventually is restricted to accepting states. Using the parlance of Section 4 , to show that every history of a program will be accepted by a $\forall$-automaton $m$, one must exhibit an invariant $I$ that satisfies obligations $\mathrm{O} 1$ and 02 and variant function $v$ satisfying

MP1: For all $x, y \in J S(\mathscr{P}, \Pi), x \rightarrow y \Rightarrow v(y) \leq v(x)$; and

MP2: For all $x, y \in J S(P, \mathrm{II}),\left(x \rightarrow y \wedge x^{(1)} \notin A_{1}\right) \Rightarrow v(y)<v(x)$.

The $\forall$-automaton for a property is isomorphic to the Buchi automaton for the negation of that property. ${ }^{11}$ This suggests there might be a connection between the proof obligations that are obtained from a $\forall$-automaton for the negation of a deterministic property and the proof obligations we obtain for a clause with a single negated property. And there is. Since there are no positive joint states with a single negated property, O3 and MP1 are equivalent. We can choose the candidate function such that 04 and MP2 are equivalent-define $u$ to be empty whenever the Buchi automaton is in an accepting state and to be $\{1\}$ when it is

\footnotetext{
${ }^{10}$ We are ignoring the behavior of $\forall$-automata on finite sequences, the placement of transition predicates in states rather than on edges, and $\forall$-automata with recurrent states (which are shown to be equivalent $\forall$-automata without such states in [26]).

${ }^{11}$ To obtain a $\forall$-automaton for $\mathscr{P}$ from a Buchi automaton for $\neg \mathscr{P}$, exchange the accepting and nonaccepting states.
} 
not. Therefore, the two techniques yield the same proof obligations for a property whose negation can be specified by a deterministic Buchi automaton.

The key insight underlying the approach in [26] is that proof obligations for the negation of a property can be extracted directly from a Buchi automaton for the property-whether or not this automaton is deterministic. ${ }^{12}$ Given a property specified by a Buchi automaton, to extract proof obligations using the approach in [26], the Buchi automaton for the negation of the property is constructed. With the technique presented in this paper, the property is decomposed into a Boolean combination of properties where the nonnegated terms must be specified by deterministic Buchi automata. Depending on the property, one or the other approach may be easier. An added advantage of our Boolean decomposition approach is that parts of the proof may be reusable since other properties might be constructed from these parts.

A final insight into the difference between the approach in [26] and our earlier approach from [2] is obtained by considering clauses of the form $\mathscr{N} \Rightarrow \mathscr{M}$, as was done in Section 4.3. The technique used in [2] is a restriction to the special case true $\Rightarrow \mathscr{M}$, and the technique used in [26] treats the other special case, $\mathscr{N} \Rightarrow$ false. Of these "special cases," the second is general; the first is not.

\section{SUMMARY}

We have described an approach to proving temporal properties of concurrent programs. This approach is based on using deterministic Buchi automata to specify properties. Such automata are quite expressive-any temporal property can be formulated as a Boolean combination of properties specified by them. Proof obligations for a property are extracted directly from the automata for that property. These proof obligations are discharged by devising suitable proof instruments. The adequacy of the proof instruments is established by verifying predicate logic formulas and triples. Thus, temporal inference is not necessary for proving temporal properties. The same techniques that prove total correctness of sequential programs can prove arbitrary temporal properties of concurrent ones.

\section{ACKNOWLEDGMENTS}

D. Gries, N. Klarlund, L. Lamport, P. Panangaden, and A. Pnueli made helpful comments on an earlier draft of this paper. We are also grateful to all three referees, who provided extremely useful criticisms on the two earlier versions of this paper. Discussions with Stavros Cosmadakis and Sheryl Brady helped in formulating the completeness proof.

\section{REFERENCES}

1. AlPERn, B. Proving temporal properties of concurrent programs: A non-temporal approach. Ph.D. thesis, Dept. of Computer Science, Cornell Univ., Feb. 1986.

2. ALPERN, B., AND SCHNEIDER, F. B. Verifying temporal properties without using temporal logic. Tech. Rep. TR 85-723, Dept. of Computer Science, Cornell Univ., Dec. 1985.

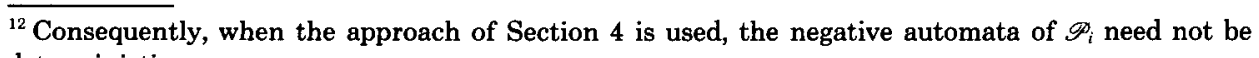
deterministic. 
3. BARRINGer, H., KUIPER, R., AND PNUEli, A. Now you may compose temporal logic specifications. In Proceedings of the 16th Annual Symposium on Theory of Computing (Washington, D.C., Apr. 1984). ACM, New York, 1984, pp. 51-63.

4. Clarke, E. M., AND Grumberg, 0 . Avoiding the state explosion problem in temporal logic model checking algorithms. In Proceedings of the 6th ACM SIGACT-SIGOPS Symposium on Principles of Distributed Computing (Vancouver, B.C., Canada, Aug., 1987). ACM, New York, 1987, pp. 294-303.

5. Clarke, E. M., Emerson, E. A., and Sistla, A. P. Automatic verification of finite-state concurrent systems using temporal logic specifications: A practical approach. In Proceedings of the 10th ACM Symposium on Principles of Programming Languages (Austin, Tex., Jan. 1983). ACM, New York, 1983, pp. 117-126.

6. Eilenberg, S. Automata, Languages and Machines, Vol A. Academic Press, New York, 1974.

7. EMERSON, E. A., AND Lei, C.-L. Modalities for model checking: Branching time strikes back. In Proceedings of the 12th ACM Symposium on Principles of Programming Languages (New Orleans, La., Jan. 1985). ACM, New York, 1985, pp. 84-96.

8. Emerson, E. A., ANv Sist'LA, A. P. Deciding branching time logic: A triple exponential decision procedure for CTL*. In Logics of Programs. Lecture Notes in Computer Science, vol. 164. SpringerVerlag, Berlin, 1983, pp. 176-192.

9. Francez, N. Fairness. Texts and Monographs in Computer Science. Springer-Verlag, Berlin, 1986.

10. Gerth, R. Transition logic. In Proceedings of the 16th Annual Symposium on Theory of Computing (Washington, D.C., Apr. 1984). ACM, New York, 1984, pp. 39-50.

11. HoARE, C. A. R. An axiomatic basis for computer programming. Commun. ACM 12, 10 (Oct. 1969), 576-580.

12. JONES, C. B. Specification and design of (parallel) programs. In Information Processing '83, R. E. A. Mason, Ed. North-Holland, Amsterdam, 1983, pp. 321-332.

13. Kurshan, R. Complementing deterministic Buchi automata in polynomial time. J. Comput. Syst. Sci. 35, 1 (Aug. 1987), 59-71.

14. KURSHAN, R. Reducibility in analysis of coordination. In Discrete Event Systems: Models and Applications, Lecture Notes in Control and Information Sciences. IIASA, vol. 103, Springer-Verlag, New York, 1987, pp. 19-39.

15. LAMPORT, L. Specifying concurrent program modules. ACM Trans. Program. Lang. Syst. 6, 2 (Apr. 1983), 190-222.

16. LAMPORT, L. What good is temporal logic. In Information Processing '83, R. E. A. Mason, Ed. North-Holland, Amsterdam, 1983, pp. 657-668.

17. LAMPORT, L., AND SChNeIDER, F. B. The "Hoare logic" of CSP, and all that. ACM Trans. Program. Lang. Syst. 6, 2 (Apr. 1984), 281-296.

18. Lehmann, D., Pnueli, A., And Stavi, J. Impartiality, justice and fairness: The ethics of concurrent termination. In Automata, Languages, and Programming. Lecture Notes in Computer Science, vol. 115. Springer-Verlag, Berlin, 1981, pp. 264-277.

19. Lichtenstein, O., AND PNUELI, A. Checking that finite state concurrent programs satisfy their linear specification. In Proceedings of the 12th ACM Symposium on Principles of Programming Languages (New Orleans, La., Jan. 1985). ACM, New York, 1985, pp. 97-107.

20. Manna, Z., AND PNUeli, A. The modal logic of programs. In Proceedings of the 6th International Colloquium on Automata, Languages, and Programming. Lecture Notes in Computer Science, vol. 71. Springer-Verlag, Berlin, 1979, pp. 385-409.

21. MANNA, Z., AND PNUELI, A. Verification of concurrent programs: The temporal framework. In The Correctness Problem in Computer Science, R. S. Boyer and J. S. Moore, Eds. International Lecture Series in Computer Science. Academic Press, London, 1981, pp. 141-154.

22. MANNA, Z., AND PNUELI, A. Verification of concurrent programs: Temporal proof principles. In Logic of Programs. Lecture Notes in Computer Science, vol. 131. Springer-Verlag, Berlin, 1981, pp. 200-252.

23. Manna, Z., AND PNUeli, A. How to cook a temporal proof system for your pet language. In Proceedings of the Symposium on Principles of Programming Languages (Austin, Tex., Jan. 1983). ACM, New York, 1983, pp. 141-154.

24. MANNA, Z., AND PNUEli, A. Verification of concurrent programs: A temporal proof system. In Foundations of Computer Science IV, Distributed Systems: Part 2, J. W. DeBakkar and J. Van Leuwen, Eds. Mathematical Centre Tracts 159, Amsterdam, 1983, pp. 163-255. 
25. Manna, Z., AND PNueli, A. Adequate proof principles for invariance and liveness properties of concurrent programs. Sci. Comput. Program. 4, 3 (1984), 257-289.

26. Manna, Z., AND PNUeli, A. Specification and verification of concurrent programs by $\forall$ automata. In Proceedings of the 14th Symposium Principles of Programming Languages (Munich, Jan. 1987). ACM, New York, 1987, pp. 1-12.

27. Misra, J., Chandy, K. M., AND SMith, T. Proving safety and liveness of communicating processes with examples. In Proceedings of the ACM SIGACT-SIGOPS Symposium on Principles of Distributed Computing (Ottawa, Canada, Aug. 1982). ACM, New York, 1982, pp. 157-164.

28. NGUYen, V., Gries, D., AND OwICKI, S. A model and temporal proof system for networks of processes. In Proceedings of the 12th ACM Symposium on Principles of Programming Languages (New Orleans, La., Jan. 1985). ACM, New York, 1985, pp. 121-131.

29. OWICKI, S. S., AND LAMPoRT, L. Proving liveness properties of concurrent programs. ACM Trans. Program. Lang. Syst. 4, 3 (July 1982), 455-496.

30. Peterson, G. L. Myths about the mutual exclusion problem. Inf. Process. Lett. 12, 3 (June $1981), 115-116$.

31. PNUeli, A. The temporal logic of programs. In Proceedings of the 18th Symposium on the Foundations of Computer Science (Providence, R.I., Nov. 1977). IEEE, New York, 1977, pp. 46-57.

32. Sistla, A. P., ANd German, S. M. Reasoning with many processes. In Proceedings of the Symposium on Logic in Computer Science (Ithaca, N.Y., June 1987). IEEE, New York, 1987, pp. 138-152.

33. STARK, E. W. Foundations of a theory of specification for distributed systems. MIT/LCS/TR342, Ph.D. thesis, MIT Laboratory for Computer Science, Cambridge, Mass., Aug. 1984.

34. VARDI, M. Y., AND WOLPER, P. An automata-theoretic approach to automatic program verification. In Proceedings of the Symposium on Logic in Computer Science (Boston, Mass, June 1986). IEEE, New York, 1986, pp. 332-344.

35. WolPER, P. Temporal logic can be more expressive. Inf. Control 56, 1-2 (1983), 72-99.

Received January 1986; revised July 1987 and July 1988; accepted July 1988 